\title{
Pharmaciana
}

Vol.10, No.2, July 2020, Page. $211-218$

ISSN: 2088 4559; e-ISSN: 24770256

DOI: $10.12928 /$ pharmaciana.v10i2.13514

\section{The effect of particle size on dissolution rate of fast dissolving oral film containing diclofenac sodium}

\author{
Fitrianti Darusman*1, Nyayu Ista Yulita ${ }^{2}$, Gita Cahya Eka Darma ${ }^{3}$ \\ Pharmacy Department, Faculty of Mathematics and Natural Sciences \\ Universitas Islam Bandung \\ Jl. Tamansari No.1 Bandung 40116, West Java, Indonesia
}

Submitted: 04-03- 2020

Reviewed: 29-03-2020

Accepted: 23-06-2020

\begin{abstract}
Diclofenac sodium is a Non-Steroidal Anti Inflammatory Drugs that if being taken orally have the side effects of peptic ulcers and undergone the first pass metabolism, and also included in the Biopharmaceutics Classification System class 2 which resulted in the low rate of dissolution. This study aims to determine the influence of particle size reduction on the dissolution rate of diclofenac sodium in the form of an FDOF dosage. The formation of diclofenac sodium nanoparticles is carried out by ionic gelation method using chitosan and sodium tripolyphosphate as a crosslinker in various ratios characterized by Particle Size Analyzer and Scanning Electron Microscopy, then it is incorporated into the form of an FDOF that were prepared by solvent casting method at a dose of 12.5 mg using variations concentration of SSG as superdisintegrant and PEG 400 as plasticizer. From the research results, diclofenac sodium nanoparticles are formed in the ratio of chitosan-sodium tripolyphosphate 6:1, have a size of $804 \mathrm{~nm}$ and spherical-shaped. The best FDOF dosage formula is F8 containing HPMC E5 LV 35\% as the film forming agent, SSG 8\% as superdisintegrant and PEG $40010 \%$ as plasticizer. FDOF formula containing diclofenac sodium nanoparticles has a slightly bitter taste, disintegration time less than one minute, surface $\mathrm{pH}$ around 7 (neutral), drug content that meets the requirements of the range of determination which is $93.24 \pm 0.96$, the cumulative amount of drug dissolved in the 28th minute is higher by $88.45 \%$ compared to FDOF containing diclofenac sodium raw material, which is only $70.0 \%$.
\end{abstract}

Keywords : diclofenac sodium, nanoparticle, ionic gelation, fast dissolving oral film, dissolution rate

\author{
*Corresponding author: \\ Fitrianti Darusman \\ Pharmacy Department, Faculty of Mathematics and Natural Sciences \\ Universitas Islam Bandung \\ Jl. Tamansari No.1 Bandung 40116, West Java, Indonesia \\ Email: efit.bien@gmail.com
}




\section{INTRODUCTION}

Diclofenac sodium is a Non-Steroidal Anti inflammatory Drugs that if being taken orally have the side effects of peptic ulcers and undergone the first pass metabolism so that only $50 \%$ of active substances can achieve systemic circulation (Katzung et al., 2012; Sweetman, 2009) In general diclofenac sodium is available in the form of conventional oral tablets. One strategy to overcome the problems above is through the development of solid intraoral film-shaped dosage form that is a Fast Dissolving Oral Film (FDOF). The delivery system in this dosage form is when the film is placed on the tongue, it immediately dissolves without the help of drinking water, then the drug can be immediately released and dissolved in the saliva and directly absorbed through the oral cavity (pregastric) to systemic circulation, so as to increase the patient's accessibility, especially those who have pathological history of peptic ulcers and swallowing difficulty (Ali et al., 2016; Ghorwade et al., 2011).

Based on the Biopharmaceutical Classification System, diclofenac sodium belongs to class II, which is a drug that has low solubility but high permeability (Karam, 2015; Wagh and Patel, 2010) and slightly difficult to dissolve in water (Anonymous, 2014, 2017). This leads to a low bioavailability of diclofenac sodium. Bioavailability is often associated with the rate of dissolution, a drug that has a low dissolution rate can result in a decrease in absorption rate especially in the gastrointestinal tract. It is a major challenge to be able to formulate diclofenac sodium into an FDOF dosage form due to the limitation of saliva volume to be able to dissolve a particular amount of drugs and the limitations of the absorption surface area in the intraoral cavity.

Theoretically, dissolution rate can be explained with the Noyes - Whitney Equation 1, as follows:

$$
\frac{d C}{d t}=k \cdot \frac{D \cdot A}{v \cdot h}(C s-C t)
$$

Equation 1 indicates that the increase in dissolution rate will occur significantly when the surface area (A) and the saturated solubility (Cs) of the active substance are increased. The surface area depends on the size of the particle, the smaller the particle size will make the contact surface area towards solvent medium to be even larger (Davies and Amino, 1993). One effort to improve both is by forming nanoparticles. Nanoparticles according to the pharmaceutical field are drug compounds which in a certain way are made in nanometer size called nanocrystal or drug compounds encapsulated in a nanometer-sized carrier system called nanocarrier (Patravale et al.,2004). Nanoparticles aim to overcome the solubility of active substances that are insoluble, improving poor bioavailability, modifying drug delivery systems so that drugs can go directly to specific areas, increasing the stability of active substances from environmental degradation (enzymatic decomposition, oxidation, hydrolysis), improving the absorption of a macromolecular compounds, reducing the bitter taste and irritating effect of an active substances in the digestive tract (Mohanraj and Chen, 2007).

In this study, the aim is to determine the effect of reduced diclofenac sodium particle size resulting from the formation of nanoparticles using the ionic gelation method, a method that utilizes ionic interactions between polycation and polyanion to form a nano-sized polyelectrolyte complex (Yeo and Kinam, 2007), which is then incorporated into an FDOF dosage form. Then the FDOF profile containing diclofenac sodium nanoparticles was compared to the FDOF containing diclofenac sodium raw material as an active ingredient based on the dissolution rate test results.

\section{MATERIALS AND METHOD Materials}

Diclofenac sodium (ex PT. Kimia Farma, Indonesia), hydroxypropylmethyl cellulose E5-LV (Wuhan Senwayer Century Chemical, China), Polyethylene glycol 400 and sodium starch glycolate 
(Gujarat Overseas Inc., India), aspartame, menthol, chitosan, tripolyphoshate sodium, acetic acid glacial and ethanol (Sigma-aldrich, United State). Other chemicals and reagents were of analytical reagent grade.

\section{Methods}

Manufacture of diclofenac sodium nanoparticle

Diclofenac sodium nanoparticles were made by ionic gelation using chitosan and sodium tripolyphosphate as nanoparticles forming agents in the ratio of 2:1, 3:1, 4:1, 5:1, 6:1 and 7:1.

Preparation of chitosan solution

Weighed chitosan was dissolved in $1 \%$ glacial acetic acid solution.

Preparation of tripolyphosphate sodium solution

Weighed sodium tripolyphosphate was dissolved in $20 \mathrm{~mL}$ demineralized aqua

Preparation of nanoparticle

Diclofenac sodium weighed as much as $100 \mathrm{mg}$ was dissolved in chitosan solution. Subsequently, the sodium tripolyphosphate solution was dropped into the mixture at room temperature $\left(28 \pm 2^{\circ} \mathrm{C}\right)$ under the rotation of the ultra-turrax homogenizer at a speed of $20000 \mathrm{rpm}$ for 5 minutes and sonicated for 5 minutes until a nanoparticle suspension was formed. The nanoparticles obtained were frozen with liquid nitrogen and lyophilized for 12 hours to obtain dry diclofenac sodium nanoparticles using a freeze dryer (Bhumkar and Pokharkar, 2006).

\section{Characterization of diclofenac sodium nanoparticle}

Particle Size Analyzer (PSA)

Determination of particle size is carried out using a particle size analyzer (PSA) by dispersing diclofenac sodium nanoparticles in aquadest at a ratio of 1/100 (v/v).

\section{Scanning Electron Microscopy (SEM)}

The characterization of nanoparticles using SEM aims to see the morphology of the diclofenac sodium nanoparticles produced.

\section{Formulation and evaluation of FDOF}

Formula optimization of FDOF

Base formula orientation was carried out to determine the optimum FDOF formula with the solvent casting method (Kalyan and Bansal, 2012) using variations in the concentration of SSG as a superdisintegrant and PEG 400 as a plasticizer to produce the best formula based on evaluating the weight, thickness and disintegration time (Table 1).

Table 1. Determination of the optimum FDOF formula

\begin{tabular}{cccccccccc}
\hline \multirow{2}{*}{ Ingredients } & \multicolumn{8}{c}{$\begin{array}{c}\text { Formula } \\
(\boldsymbol{\%})\end{array}$} \\
\cline { 2 - 11 } HPMC E5-LV & F1 & F2 & F3 & F4 & F5 & F6 & F7 & F8 & F9 \\
\cline { 2 - 11 } SSG & 35 & 35 & 35 & 35 & 35 & 35 & 35 & 35 & 35 \\
PEG-400 & 5 & 4 & 4 & 6 & 6 & 6 & 8 & 8 & 8 \\
Aspartame & 2.5 & 2.5 & 2.5 & 2.5 & 2.5 & 2.5 & 2.5 & 2.5 & 2.5 \\
Menthol & 10 & 10 & 10 & 10 & 10 & 10 & 10 & 10 & 10 \\
\hline
\end{tabular}


Manufacture of FDOF

The optimum base formula based on evaluation results was chosen to make FDOF preparations containing diclofenac sodium raw material and diclofenac sodium nanoparticles each at a dose (equivalent) of $12.5 \mathrm{mg}$ (Table 2).

Table 2. FDOF formula

\begin{tabular}{ccc}
\hline \multirow{2}{*}{ Ingredients } & \multicolumn{2}{c}{ Formula } \\
\cline { 2 - 3 } & F8-A & F8-B \\
\hline HPMC E5-LV (\%) & 35 & 35 \\
SSG (\%) & 8 & 8 \\
PEG-400 (\%) & 10 & 10 \\
Aspartame (\%) & 2.5 & 2.5 \\
Menthol (\%) & 10 & 10 \\
Diclofenac sodium (mg) & 12.5 & - \\
Diclofenac sodium nanoparticle (mg) & - & 12,5 \\
\hline
\end{tabular}

Evaluation of FDOF

Evaluation of the final preparation includes organoleptic, weight diversity, thickness, disintegration time, surface $\mathrm{pH}$, drug content and dissolution rate test.

\section{RESULT AND DISCUSSION}

\section{Manufacture of diclofenac sodium nanoparticle}

In the manufacture of diclofenac sodium nanoparticles that did not experience precipitation occurred at a chitosan-sodium tripolyphosphate crosslinking ratio of 6:1. Precipitation that occur indicates a larger particle size. Precipitation is caused by interactions between chitosan and sodium tripolyphosphate which have reached stoichiometry, causing more crosslinking to form between them and produce insoluble complexes.

\section{Characterization of diclofenac sodium nanoparticle}

Particle Size Analyzer (PSA)

The results of the characterization of nanoparticles using PSA showed a sample with a particle size of $804 \mathrm{~nm}$, where the size of the nanoparticles ranged from 10 to $1000 \mathrm{~nm}$ (Nagarajan et al., 2015) (Figure 1).

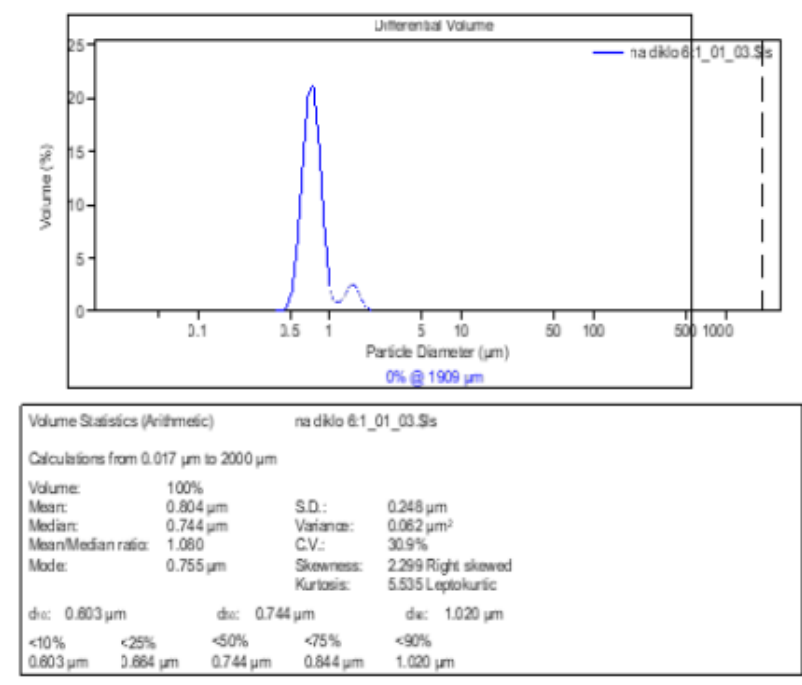

Figure 1. Particle size analyzer result of diclofenac sodium nanoparticle 
Scanning Electron Microscopy (SEM)

Characterization using SEM aims to see the morphology of the nanoparticles produced. SEM is used to observe grain boundaries, distribution, phase limits, evidence of mechanical deformation and chemical compositions that have magnifications up to 200.000x, can observe thicknesses from $200 \AA$ to $0.5 \mu \mathrm{m}$ (Ruíz,2015). The evaluation results show that diclofenac sodium nanoparticles have a spherical shape and tend to be homogeneous compared to pure diclofenac sodium observed at $800 \mathrm{x}$ magnification with particle sizes ranging from 500-800 nm (Figure 2).
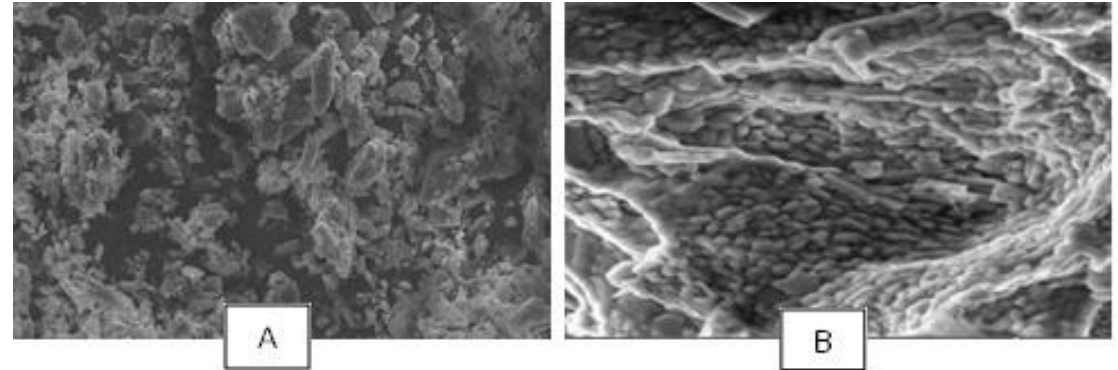

Figure 2. Morphology of powder by Scanning Electron Microscope (SEM) with 800x magnification : A. Pure diclofenac sodium; B. Diclofenac Sodium Nanoparticles

\section{Formulation and evaluation of FDOF}

Optimization of FDOF formula

Formula optimization is carried out to select and determine the best base for film formation from the aspect of diversity in weight, thickness and disintegration time. Based on the evaluation results of the formula optimization, F8 is the best formula because it has a coefficient of variance in the diversity of weights, thickness and disintegration time that is smaller than other formulas, so it can be concluded that F8 can provide films with good characteristics (Table 3).

Table 3. Evaluation of the FDOF formula optimization

\begin{tabular}{lccc}
\hline \multirow{2}{*}{ Formula } & \multicolumn{3}{c}{ Evaluation } \\
\cline { 2 - 4 } & $\begin{array}{c}\text { Weight Diversity } \\
(\mathbf{m g})\end{array}$ & $\begin{array}{c}\text { Thickness } \\
(\mathbf{m m})\end{array}$ & $\begin{array}{c}\text { Disintegration Time } \\
\text { (second) }\end{array}$ \\
\hline F1 & $41.586 \pm 0.041$ & $0.114 \pm 0.00$ & $57.44 \pm 1.51$ \\
F2 & $42.093 \pm 0.075$ & $0.114 \pm 0.00$ & $66.26 \pm 3.94$ \\
F3 & $42.963 \pm 0.387$ & $0.114 \pm 0.00$ & $85.21 \pm 5,61$ \\
F4 & $41.467 \pm 0.005$ & $0.114 \pm 0.00$ & $46.31 \pm 0.56$ \\
F5 & $42.383 \pm 0.015$ & $0.114 \pm 0.00$ & $57.36 \pm 0.45$ \\
F6 & $43.487 \pm 0.032$ & $0.114 \pm 0.00$ & $53.55 \pm 0.48$ \\
F7 & $42.003 \pm 0.184$ & $0.114 \pm 0.00$ & $32.58 \pm 2.44$ \\
F8 & $42.820 \pm 0.125$ & $0.114 \pm 0.00$ & $43.05 \pm 0.40$ \\
F9 & $43.103 \pm 0.005$ & $0.114 \pm 0.00$ & $53.39 \pm 0.94$ \\
\hline
\end{tabular}

Manufacture of FDOF

After getting the best formula from the optimization results, two FDOF formulations were prepared, namely FDOF containing diclofenac sodium raw material (F8-A) and FDOF containing diclofenac sodium nanoparticles (F8-B) with each dose (equivalent) $12.5 \mathrm{mg}$.

Final Evaluation of FDOF

Organoleptic evaluation results, F8-B has a transparent colour, odorless and has a slightly bitter taste, better than F8-A which has an slightly transparent, odorless and bitter taste. This proves that diclofenac sodium nanoparticles can dissolve well when incorporated in a hydrophilic FDOF base and also improving its bitter taste (Table 4). 
Table 4. Organoleptic evaluation of FDOF

\begin{tabular}{ccccc}
\hline & \multicolumn{4}{c}{ Organoleptic } \\
\cline { 2 - 5 } Formula & Shape & Colour & Odor & Taste \\
\cline { 2 - 4 } F8-A & Square & Slightly transparent & Odor & Bitter \\
F8-B & Square & Transparent & Odorless & Slightly bitter \\
\hline
\end{tabular}

The results of testing the diversity of weights, thickness, disintegration time and surface $\mathrm{pH}$ in both FDOF preparations fulfilled the requirements (Table 5). Both F8-A and F8-B have a disintegration time less than 1 minute and a surface $\mathrm{pH}$ value of close to 7 (neutral). It is known FDOF with a basic or acidic $\mathrm{pH}$ can irritate the oral cavity when used so that it affects the comfort of the patients. This proves that the formation of nanoparticles in diclofenac sodium does not affect the degree of acidity and basicity.

Table 5. Evaluation of FDOF

\begin{tabular}{cccccc}
\hline Formula & \multicolumn{5}{c}{ Evaluation } \\
\cline { 2 - 6 } & $\begin{array}{c}\text { Weight Diversity } \\
(\mathbf{m g})\end{array}$ & $\begin{array}{c}\text { Thickness } \\
(\mathbf{m g})\end{array}$ & $\begin{array}{c}\text { Disintegration Time } \\
\text { (second) }\end{array}$ & Surface pH & $\begin{array}{c}\text { Drug content } \\
(\boldsymbol{\%})\end{array}$ \\
\cline { 2 - 6 } F8-A & $42.7 \pm 0.5$ & $0.116 \pm 0.003$ & $34.50 \pm 0.51$ & $6.828 \pm 0.12$ & $120.3 \pm 3.81$ \\
F8-B & $43.7 \pm 0.9$ & $0.111 \pm 0.002$ & $33.60 \pm 0.92$ & $6.952 \pm 0.05$ & $93.24 \pm 0.96$ \\
\hline
\end{tabular}

The drug content of F8-A was $120.3 \pm 3.81$ and F8-B was $93.24 \pm 0.96$ (Table 5). When referring to the requirements of diclofenac sodium content in the preparation that is equal to $90-110 \%$ with an SD value of not more than 6 (Anonymous, 2014; Irfan et al., 2016) then F8-B meets the requirements, while F8-A does not meet the requirements. F8-B meets the requirements because the active ingredient is diclofenac sodium in the form of nanoparticles so that the solubility in the water increases, the homogenization process becomes easier and faster in an FDOF base. F8-A is not eligible because diclofenac sodium solubility is rather insoluble in water and forms clumps that are difficult to be homogenized in an FDOF base, thereby affecting its distribution in the preparation.

From the dissolution rate test results in the $28^{\text {th }}$ minute, it was proven that FDOF containing diclofenac sodium nanoparticles had a cumulative amount of dissolved drug which was higher at 88.45\% compared to FDOF containing diclofenac sodium raw material, which was only $70.0 \%$ (Figure 3). This is consistent with Noyes and Whitney's equations that an increase in the affinity of the system is due to an increase in the contact surface area at the same amount (Adeyeye at el, 2007).

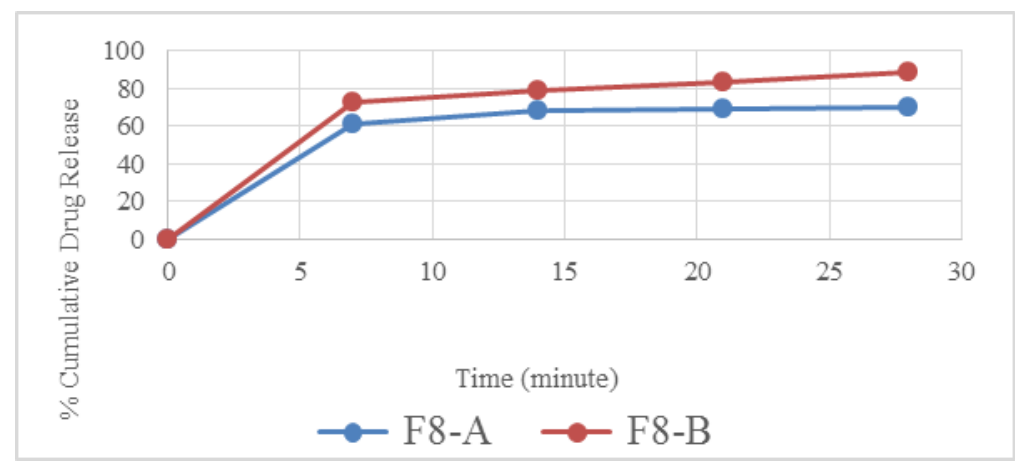

Figure 3. Curve ratio of the amount of dissolved substances to the time between FDOF containing diclofenac sodium raw material (F8-A) against FDOF containing diclofenac sodium nanoparticles (F8-B) 


\section{CONCLUSION}

From the results of this study can concluded that diclofenac sodium nanoparticles were made by ionic gelation method with chitosan and sodium tripolyphosphate as a crosslinker at a ratio of 6: 1 could produce a nanoparticles of $804 \mathrm{~nm}$ in size and spherical in shape. The best FDOF preparation formula is F8 containing HPMC E5 LV 35\% as a film forming agent, SSG 8\% as a superdisintegrant and $10 \%$ PEG 400 as a plasticizer. FDOF formula containing diclofenac sodium nanoparticles has a slightly bitter taste, disintegration time is less than one minute, surface $\mathrm{pH}$ is around 7 (neutral), drug content that meets the requirements of the range of determination which is $93.24 \pm 0.96$, the cumulative amount of drug dissolved in the $28^{\text {th }}$ minute is higher by $88.45 \%$ compared to FDOF containing diclofenac sodium raw material, which is only $70.0 \%$.

\section{ACKNOWLEDGEMENT}

The authors are thankful to PT. Kimia Farma, Jakarta, Indonesia for generously giving the samples of diclofenac sodium. We are also thankful to the Principal and Management UNISBA of Pharmacy, Bandung, Indonesia, for his constant encouragement.

\section{REFERENCES}

Adeyeye, C. M., Li, P., \& Chemistry, P. (1990). Diclofenac Sodium: Analytical Profiles of Drug Substances, Florey, K., 19, 123-144.

Ali MS, Vijendar, C., D, S. K., \& Krishnaveni, J. (2016). Formulation and evaluation of fast dissolving oral films of diazepam. Journal of Pharmacovigilance, 4(3). https://doi.org/10.4172/2329-6887.1000210.

Anonymous. (2014). Farmakope Indonesia V (Vol. 2). Indonesia: BPOM RI.

Anonymous. (2017). Combined Index to USP 41 and NF 36, 1-5, 1-72.

Bhumkar, D. R., \& Pokharkar, V. B. (2006). Studies on Effect of pH on Cross-linking of Chitosan With Sodium Tripolyphosphate: A Technical Note, 7(2), 2-7.

Buzea, C., Blandino, I. I. P., \& Robbie, K. (2007). Nanomaterials and nanoparticles: Sources and toxicity, 2(4), 1-103.

Davies, T. F., \& Amino, N. (1993). A new classification for human autoimmune thyroid disease. Thyroid (Vol. 3). https://doi.org/10.1089/thy.1993.3.331.

Ghorwade, V., Patil, A., Patil, S., Ikkurthi, K., Inuganti, K. S., \& Porandla, V. (2011). Formulation and evaluation of Montelukast sodium fast dissolving films by using Gelatin as a film base. Research Journal of Pharmaceutical, Biological and Chemical Sciences, 2(3), 880-888.

Irfan, M., Rabel, S., Bukhtar, Q., Imran, M., Jabeen, F., \& Khan, A. (2016). Orally disintegrating films : A modern expansion in drug delivery system. Saudi Pharmaceutical Journal, 24(5), 537546. https://doi.org/10.1016/j.jsps.2015.02.024.

Kalyan, S., \& Bansal, M. (2012). Recent Trends in the Development of Oral dissolving Film, 4(2), $725-733$.

Karam, R. (2015). Biowaivers : criteria and requirements prepared by Dr . Mazen Kurdi, 1-11.

Katzung, B. G., Masters, S. B., \& Trevor, A. (2012). Basic dan Clinical Pharmacology (Vol. 12). https://doi.org/10.1016/S0065-7743(08)61545-6.

Mohanraj, V. J., \& Chen, Y. (2007). Nanoparticles - A review. Tropical Journal of Pharmaceutical Research, 5(1), 561-573. https://doi.org/10.4314/tjpr.v5i1.14634.

Nagarajan, E., Shanmugasundaram, P., Ravichandiran, V., Vijayalakshmi, A., Senthilnathan, B., \& Masilamani, K. (2015). Development and Evaluation of Chitosan Based Polymeric Nanoparticles of an Antiulcer Drug Lansoprazole, 5(4), 20-25. https://doi.org/10.7324/JAPS.2015.50404.

Patravale, V. B., Date, A. A., \& Kulkarni, R. M. (2004). Nanosuspensions : a promising drug delivery strategy, (1992), 827-840. https://doi.org/10.1211/0022357023691.

Ruíz, A. A. B. (2015). Nanoparticle Technology for Drug Delivery, 3(2), 54-67.

Sweetman, S. C. (2009). Martindale The Complete Drug Reference (Vol. 53). https://doi.org/10.1017/CBO9781107415324.004. 
Wagh, M. P., \& Patel, J. S. (2010). Biopharmaceutical classification system: Scientific basis for biowaiver extensions. International Journal of Pharmacy and Pharmaceutical Sciences, 2(1), $12-19$.

Yeo, Y., \& Kinam, P. (2007). Recent Advances in Microencapsulation Technology. Encyclopedia of Pharmaceutical Technology, 2315-2327. https://doi.org/10.1081/E-EPT-120028567. 